\title{
Assessing scalability of a low-voltage distribution grid co-simulation through Functional Mockup Interface
}

\author{
Vincent Reinbold ${ }^{\mathrm{a}}$ and Christina Protopapadaki ${ }^{\mathrm{b}, \mathrm{c}}$ and Jean-Philippe Tavella ${ }^{\mathrm{d}}$ and \\ Dirk Saelens ${ }^{\mathrm{b}, \mathrm{c}}$ \\ ${ }^{a}$ Group of electrical engineering - Paris, CNRS, CentraleSupélec, Univ. Paris-Sud, Université \\ Paris-Saclay, Sorbonne Université, 3 \& 11 rue Joliot-Curie, Plateau de Moulon 91192 \\ Gif-sur-Yvette CEDEX, France; 'bU Leuven, Department of Civil Engineering, Building \\ Physics Section, Kasteelpark Arenberg 40 - box 2447, BE-3001 Heverlee, Belgium; \\ ${ }^{c}$ EnergyVille, Thor Park 8310, BE-3600 Genk, Belgium; ${ }^{\text {EDDF R\&D, France }}$
}

\author{
ARTICLE HISTORY \\ Compiled December 17, 2019
}

\begin{abstract}
State-of-the-art Modelica tools for modeling and simulating multi-physical systems have reached certain maturity among the building physics community. Hence, simulation is widely used for control, sizing and performance assessment of energy systems. However, serious efficiency issues arise for large-scale models. This article proposes a practical application of co-simulation methods on detailed district energy systems. The aim of this study is to assess performance and scalability of cosimulation through Functional Mock-up Interfaces on a detailed and multi-physical district model. In particular, we propose a comparative analysis between classical simulation and co-simulation methods and a scalability analysis on a growing number of buildings. The models have been implemented using Modelica language and the OpenIDEAS library. A decomposition approach is taken for modeling the entire system, while stochasticity in the inputs is taken into account. Results are presented for various integration scenarios, including a classical integrated simulation for reference and co-simulations involving different master-algorithms within Dymola and DACCOSIM 2017. Scenarios are compared in terms of speed-up and accuracy of principal physical quantities representing key performance indicators such as indoor temperature, current and voltage at building's connection. The analysis shows that co-simulation can run up to 90 times faster than the integrated simulation for 24 buildings, while ensuring acceptable accuracy.
\end{abstract}

\section{KEYWORDS}

Co-simulation; District Energy System; low-voltage grid; Modelica; Functional Mockup Interface; Efficient Simulation; Large-scale Model

\section{Introduction}

On November 2016, European commission updated the energy efficiency policies, including a new 30\% energy efficiency target for 2030 (European Commision 2016), reinforcing the 2012's targets (European Union 2012). Specific measures and policies are directed toward all the energy chain, from production to consumers. New legislation enforces for instance: a renovation rate for public buildings, free data access on consumption 
through smart metering, increasing the share of renewable energy or promoting circular economy (by means of co-generation or recycling waste energy). In this context, District Energy Systems (DES) are at the forefront of the engineering research.

A DES consists of all components enabling production, storage and distribution of energy to the buildings of a district. It may refer to electricity, gas, heating and/or cooling networks (Carvalho et al. 2016; Frederiksen and Werner 2013). Classical DES are facing deep changes, mainly introduced by distributed renewable energy generation, highly efficient technologies or smart control such as model predictive control.

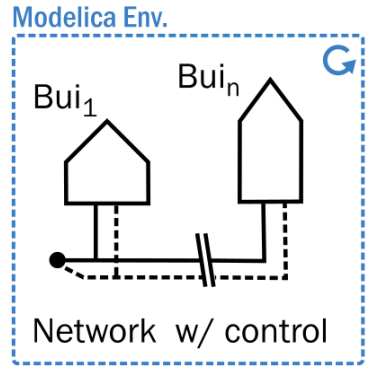

(a) Integrated simulation of the full DES. White box models or simplifications.

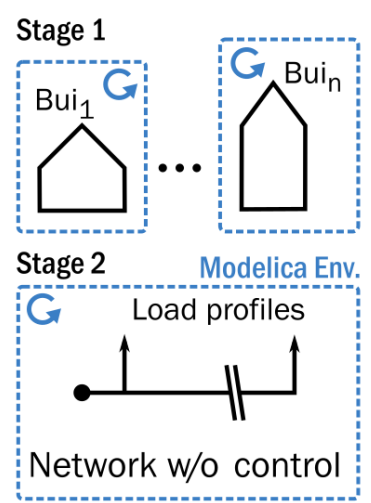

(b) Decoupled approach: Stage 1: Simulating each building independently. Stage 2: Simulating the network using load demand profiles.

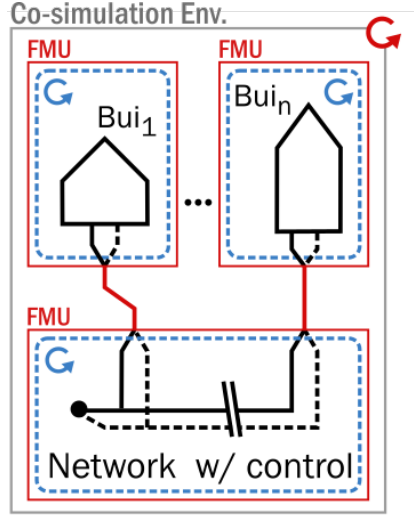

(c) Co-simulation of the DES using FMU components.

Figure 1. Representation of the 3 simulation approaches. (FMU) , $\square$ co-simulation environment, $\mathrm{G}$ classical simulation integration algorithm, $\mathrm{G}$ co-simulation masteralgorithms, — physical network connection, -...- feedback control and data communications, - FMU communications.

As a consequence, research has directed efforts on models and simulation to assess energy performance, controls and sizing of the future DES. For these systems, an integrated modeling platform is required, capable of simultaneously considering numerous heterogeneous buildings, the dispatch of energy through the network and all the communication signals related to smart meters or controls. Detailed and multi-physical simulation of a large DES is challenging. Several bottlenecks are usually encountered, such as : multi-physical phenomenons, multiple time-space scales and dynamics, implementation of controllers, stochasticity (Baetens and Saelens 2016) and interoperability. For this purpose, different modeling approaches are possible. In the following, we propose to describe two classical methodologies together with the co-simulation approach illustrated in fig. 1.

An illustration of a fully integrated approach is proposed in fig. 1(a). Regardless of the type of DES (electrical and/or thermal), all interactions between the buildings and the network are technically possible, including physical interaction, feedback control or data signals.

The paradigm of integrated modeling using detailed physics-based models (white-box models) attracted researchers' attention and has reached certain maturity on the building level, especially regarding available models and tools (Fritzson 2015). In Wetter, Bonvini, and Nouidui (2016), the authors highlight advantages of equation-based modeling languages, such as Modelica, for symbolic manipulation and multi-physical inte- 
gration. In 2017, a coordinated project entitled "New generation computational tools for building and community energy systems based on the Modelica and Functional Mockup Interface standards" came to an end, linking different contributions and developing an open-source core library called (Wetter et al. 2015). Likewise, Baetens et al. (2015) propose an open framework for district energy simulation. These libraries, fully based on the Modelica language, aim to tackle the known issues of DES such as multi-physical modeling, generation of controls and efficient code for simulation and optimisation.

The use of white box models is widely accepted in research and various validation studies demonstrated their reliability at the building level (Nouidui et al. 2012; Jensen 1995; Kersken, Heusler, and Strachan 2014). But, whereas multi-physical integration issues were circumvented by the use of open source libraries and white box models, researchers are facing other types of challenges, related to computation time and upscaling of the computational complexity. In a multi-physical context, Casella (2015) presents a detailed list of known issues with large scale models, related to compilation, memory allocation or solvers. The most important are :

- the calculus of the Jacobian matrix and the inversion of the Hessian matrix for implicit solvers, which grows as $O\left(N^{3}\right)$ with $\mathrm{N}$ the number of states,

- the memory allocation of those matrices,

- non-linear algebraic loops, between buildings and grid for instance, that requires iterative calculus,

- localized activities that impose to reduce the time steps and span the entire system, or events that requires iterative calculus of the system until convergence, and

- widely different time scales, requiring small enough steps to stay within the allowed error tolerance for the fastest dynamic.

All these issues are combined within the DES. In particular, we denote different dynamics (thermal, electrical, fluids), numerous algebraic loops, mainly due to the electrical connection between grid and buildings and local activities since the occupancy behaviour are different for each building. As a result, the use of integrated DES models for control or optimization applications may be compromised on large scale systems (Privara et al. 2013). In additions, DES simulations usually consider stochastic inputs for assessing local and general effects on a wide spectrum of scenarios multiplying the number of needed simulations and the computational cost.

To overcome this scalability issue and try to speed-up simulations, some model simplifications may be introduced. Depending on whether the focus lies on the network or building, either simplified thermal structures or an ideal grid are considered. Most of these simplified thermal structures are based on the concept of grey-box models and stochastic linear differential equations (Melgaard 1994; Madsen 2007). In this context, Kim et al. (2014) presented a reduction technique for building envelope models in urban simulations to ensure a good trade-off between computation time and precision. In 2015, Nytsch-Geusen and Kaul developed a tool that generates populations of low-order models based on geographic information and statistical methods to calculate energy demand. A similar approach of generation and validation of grey-box models can be found in (De Coninck et al. 2015). Existing tools, like CitySim, allow dynamic simulation on a urban scale. The later uses the 3D geometry of the building to derive a dynamic thermal model based on equivalent electric circuit as described in Robinson et al. (2009). It was recently compared to the Building Energy Simulation Test (BESTEST) validation procedure in (Walter and Kämpf 2015). Likewise, TEASER has been developed to generate dynamic simulation of thousands of buildings with low inputs requirement. The 
work-flow consists of several steps, including data acquisition from CityGML models for instance, data enrichment and generation of reduced order model based on AixLib or Annex 60 Modelica libraries (Müller et al. 2016; Saelens, Wetter, and van Treeck 2017).

Those approaches proved to be efficient for total energy use or thermal demand Reinhart and Davila (2016). However, due to unmodelled inputs or simplifications, a significant bias on the output still exists, and may reduce the use of such methods for control strategies, topology or sizing optimization for districts. On the building level, an assessment of white-box and grey-box models is done for model predictive control (MPC) (Zhang et al. 2015). Results show a significant difference in the energy savings between both approaches.

An alternative to building or network simplifications for simulation speed-up is to reduce interactions. As a result, a decoupled approach emerged that may consider detailed models for both district and building levels and simulates the DES in two steps. An illustration of this method is proposed in fig. 1(b). First, building loads are computed in detailed, considering stochastic occupancy profiles, heterogeneous structures, decentralized energy generation, etc. Then, the network behavior is simulated by applying the building loads to assess energy quality, such as voltage drops and thermal constraints. This last method proved to be useful for statistical analysis and energy policy assessment (Protopapadaki and Saelens 2017; Protopapadaki, Baetens, and Saelens 2015). Nevertheless, in this case, interaction between building and grid can only be one-sided, prohibiting any kind of feedback control or data communication during run-time. For instance, decentralized load control for voltage regulation is limited.

A compromise between the fully integrated and the decoupled approach is possible, insuring communication and feedback control, while the buildings and network are simulated almost independently or in parallel. This method is called co-simulation. Such approach is illustrated in fig. 1(c). It allows a coherent integration of a decomposed system, by assigning a specific solver to each sub-component, while exchanging data during run-time. The first challenge of such approach is to create tools and algorithms enabling decomposition and simulation of the different parts. Several solutions have been developed for building and district simulation, such as the BCVTB (Wetter 2011), EnergyPlus (Nouidui, Wetter, and Zuo 2014) or DACOSSIM (Galtier et al. 2015).

In 2008, the Modelisar project begun to develop an open-source and community driven standard for co-simulation and model exchange named Functional Mock-up Interface (FMI) (Blochwitz et al. 2011). Initially developed for the automotive industry, its aim was to facilitate exchange between partners and different platforms. The FMI aims to standardize and facilitate the interface for general purpose co-simulation. It is now largely used and allows exporting and importing models to and from numerous platforms. One can find an exhaustive list of compatible environments along with various literature online ${ }^{1}$.

Several building-level applications of co-simulation through FMI standards can be found in (Nouidui, Wetter, and Zuo 2014; Raad et al. 2015). On the contrary, only few applications of co-simulation at district level are available. G. Zucker et al. achieved a co-simulation of a large district heating system involving low-order buildings models in (Zucker et al. 2016). The co-simulation work-flow couples the Ptolemy II (Ptolemaeus 2014) environment to the FMI++ library (Widl et al. 2013) along with EnergyPlus for model exportation. Recently, Vialle et al. (2017) implemented the co-simulation of a district within the DACCOSIM environment to demonstrate the feasibility of such large simulation on a cluster of computers (Galtier et al. 2015).

\footnotetext{
${ }^{1}$ https://www.fmi-standard.org/
} 
Only few studies deal with co-simulation and scalability using white box district models. To fill this gap, we propose a methodological assessment of co-simulation methods in the district context (fig. 1(c)), in terms of speed-up, scalability and accuracy. The present paper proposes a co-simulation approach for DES simulation using the FMI standards and the Modelica language. The aim is twofold: First, validate this approach, by comparing it to the state-of-the-art integrated method (fig. 1(a)), and secondly, quantify and compare the scalability performance of the co-simulation (fig. 1(c)). Because the decoupled method (illustrated in fig. 1(b)) does not allow feedback control or data exchange, it is considered out of scope for the comparison in this paper.

An integrated model for DES is created for a growing number of buildings along with its adaptation to the co-simulation approach. Consequently, this study also includes practical aspects of DES co-simulation, such as decomposition, parameterization and adaptations. Particular attention will be paid to the management of resources, i.e. the weather and occupancy data, to ensure genericity and a minimum use of memory. At the same time, the present paper also considers different master-algorithms. Indeed, the latter are responsible for orchestrating of the co-simulation, and their implementations may have significant impact on the scalability results. In the scope of this article, Dymola and DACCOSIM environments will be compared. Dymola, because it is a widely used Modelica environment and DACCOSIM, because it allows more advanced implementations, such as clustering, adaptive communication steps and parallel computing.

This article is structured as follows: Methodology and tools are presented in section 2, along with a description of models, scenarios and adaptations for co-simulation purposes. Section 3 presents the comparative results in terms of accuracy and scalability. Finally, overview and conclusion are developed in section 4 .

\section{Methodology and models}

As a first assumption, we limit our study to an electrical low-voltage distribution grid and a variable number of detailed buildings. Our main results may be extended to other DES technologies in future work, such as district heating systems. Both the integrated simulation and the co-simulation approach are implemented using the same models such that any differences can only be input to the integration scheme. These core models are first described in section 2.2. Then, some adaptations on the models are presented in section 2.3 for co-simulation purposes, such as decomposition and boundary condition integration. After that, various scenarios are proposed to build the comparison and co-simulation assessment. The accuracy of each case is estimated using classical error indicators for current, voltage and room temperature profiles. Key performances indicators related to the computation time are also defined, and finally, scalability is estimated based on the speed-up and its evolution with respect to the number of buildings.

\subsection{Tools}

All models and their components are implemented using Modelica language and the IDEAS library v1.0.0. They are compiled and exported as FMU using Dymola 2017 (64bits) coupled with the Visual Studio $V C++$ express compiler. All simulations are done within Dymola while co-simulation is handled either by Dymola or by DACOSSIM. Compilation and simulation were run on a laptop composed of a dual-core i7-6600U CPU $2.8 \mathrm{GHz}$ and $8 \mathrm{~GB}$ of RAM, operated by Windows 7 Pro. 64 bits. 


\subsubsection{Modelica tools}

IDEAS is a free open-source library with models for buildings, district energy systems and control (Baetens et al. 2015). It allows a complete integration of dynamic simulation at both building and district level. Since v1.0.0, the IDEAS library fully integrates the Annex60 library which has been developed within the Annex 60, project ${ }^{2}$ of the International Energy Agency's Energy in Buildings and Communities Program (Wetter et al. 2015; Saelens, Wetter, and van Treeck 2017). The latter project is to be continued as the IBPSA Project $1^{3}$.

\subsubsection{Functional Mock-up Interface and Master Algorithm}

As stated in the introduction, the FMI is a general and independent specification for cosimulation and model exchange. It has become a state-of-the-art standard for dynamic model export and is available in numerous modeling environments. In practice, the Functional Mock-up Unit (FMU) includes as a set of C-functions composing the model, and an XML file defining internal variables, inputs, outputs and their dependencies. Sub-components are first exported as FMUs, and act like black-box models. These can be instantiated, combined, interconnected and co-simulated within a compatible environment as illustrated in fig. 1(c).

In order to take into account the co-simulation implementation in the assessment, two different co-simulation environments will be used. First, Dymola which uses a fixed step master-algorithm (MA), and secondly DACCOSIM 2017 (Galtier et al. 2015), which allows more advanced implementation such as adaptive communication step and parallel computing. Simulation scenarios and MA settings will be detailed in section 2.4.

\subsection{Building and feeder modeling}

A detailed district model using a variable number of buildings connected to an electrical feeder has been implemented. This feeder represents the reference case for simulation result comparisons. Figure 2 gives a schematic representation of the described DES model: an ideal medium voltage source is connected to a $400 \mathrm{kVA}$ transformer, feeding a three-phase grid. Buildings are alternatively connected one after the other to different electrical phases. A schematic representation of the multi-zone building models is given in fig. 2, involving thermal envelope, ventilation, occupancy behavior and heating system. The FMI adaptation block that allows the FMU exportation of the component for co-simulation will be detailed in section 2.3.1. For each building, we consider 3 thermal zones that correspond to the ground, first and second floor, and an ideal individual heat-pump (HP) with a fixed coefficient of performance (COP). The main building characteristics are summarized in table 1. For more details, one can freely access the model in IDEAS (2017). The building structure and heating system are assumed to be identical for all buildings in this study. However, to simulate load variability, heterogeneous occupancy profiles describing temperature set points, electrical and hot-water demands are used, which are derived from a stochastic model (Baetens and Saelens 2016). The electrical grid model is also available in IDEAS (IDEAS 2017) and was developed in Verbruggen et al. (2011); Baetens (2015). The cable section stays fixed regardless of the amount of buildings. More characteristics are available in table 1(b).

\footnotetext{
2 http: //www.iea-annex60.org/

3 https://ibpsa.github.io/project1/
} 


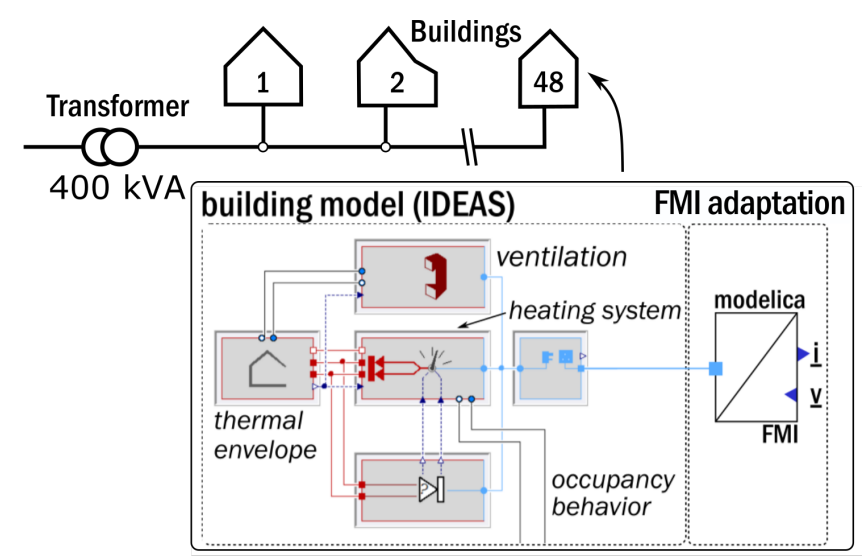

Figure 2. Representation of the district energy system model.

Table 1. Characteristic of the considered District Energy System.

(a) Building

\begin{tabular}{lrl}
\hline description & value & unit \\
\hline Number of zones & 3 & - \\
Inner surface & 77.1 & $\mathrm{~m}^{2}$ \\
Windows to wall ratio & 0.15 & - \\
Glazing U-value & 1.0 & $\mathrm{~W} /\left(\mathrm{m}^{2} . K\right)$ \\
Glazing g-value & 0.598 & - \\
Wall U-value & 0.30 & $\mathrm{~W} /\left(\mathrm{m}^{2} . K\right)$ \\
\hline HP nominal power & 15 & $\mathrm{~kW}$ \\
COP & 3.0 & - \\
\hline
\end{tabular}

(b) Grid

\begin{tabular}{lrl}
\hline description & value & unit \\
\hline Cable Resistance & 0.461 & $\mathrm{~m} \Omega . \mathrm{m}^{-1}$ \\
Cable Reactance & 0.0723 & $\mathrm{~m} \Omega . \mathrm{m}^{-1}$ \\
Cable Section & 70 & $\mathrm{~mm}^{2}$ \\
Cable Nominal current & 125 & $\mathrm{~A}$ \\
Transformer Nominal & 400 & $\mathrm{kVA}$ \\
Power & & \\
\hline
\end{tabular}

\subsection{Adaptation for co-simulation}

\subsubsection{Decomposition}

Co-simulation of the DES is based on an a priori decomposition of the system which allows simulators to be executed while exchanging data for a coherent integration. This decomposition can have a significant impact on the performance of the integration, and it attempts to achieve the following:

- privilege the exchange of slow quantities to maximize the communication time step,

- adapt specific solvers to sub-components in order to improve and speed-up each integration,

- break algebraic loops to make sub-models simpler to solve, and

- create equivalent-size sub-systems to optimize the CPU usage in case of parallel or cluster computing.

Consequently, a large amount of possible combinations for decomposition exists. To our knowledge, there is no method or tools to optimize such problem. Additionally, it depends on the case and relies on the number of states, the incidence matrix and the hardware architecture of the computer or the computer cluster that will run the co-simulation. However, the use of Modelica language usually makes the decomposition straightforward, since it is a component-oriented modeling language that explicitly declares decomposition, inputs, outputs and ports of each sub-component. Moreover, 
DES typically consist of many similar components that are repeated and linked to each other. As a consequence, one could consider creating communications between clusters of buildings, individual buildings or, deeper, between each heating system, thermal envelope, and the grid. As a starting point, the grid and each building were treated as separate FMUs as illustrated in fig. 1(c). The interest is twofold: first, it breaks the algebraic loop that may occur between the current and the voltage in the electrical connection and secondly, it creates equivalent size sub-systems since all buildings are similar. Other finer decomposition schemes, for instance, between the thermal structure, the HVAC of each building and the grid, could be considered in future work. The benefit of such a decomposition would be to decouple the rather slow thermal response of the building envelope and the heating system, which might involve fast dynamics or numerous events.

FMI specifications do not support all the Modelica interfaces, especially flow/effort connectors, such as electrical, fluid or heat ports. As a result, some modifications are needed to comply to the standards concerning inputs and outputs of sub-components. This can be achieved by adding adaptation blocks that translate flow/effort to classical block connectors. An example for the electrical connector is proposed in code 1, where the connector Modelica.Electrical.QuasiStationary.SinglePhase.Interfaces.PositivePin is converted to tree connectors using the Modelica standard library: Modelica.ComplexBlocks.I nterfaces.ComplexOutput, Modelica.ComplexBlocks.Interfaces.ComplexInput and Modelica. Blocks.Interfaces. Reallnput. Note that this adapter imposes causality in order to comply with the FMI specification: the current is imposed as an output whereas frequency and voltage are inputs. It is a flow source and can be adapted to the building outputs for instance (c.f. fig. 2). An analogue block was also developed for the network with an opposite causality, i.e. imposing frequency and voltage and getting current as input.

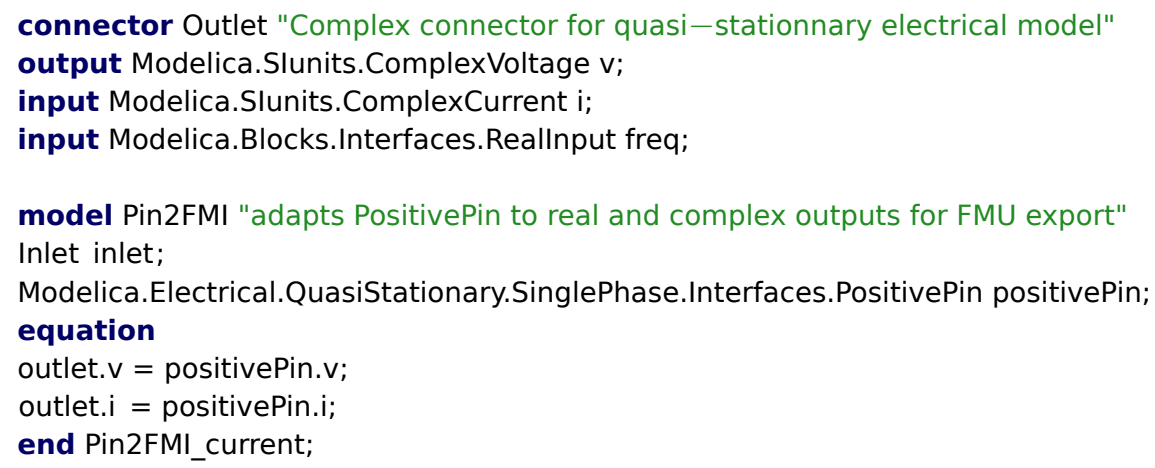

Code 1: Modelica model of electrical adapter for FMU export. Exposing the current as complex output and frequency and voltage to real input and complex input respectively.

\subsubsection{Handling boundary conditions}

Concerning external resources, weather data should be delegated to a single FMU because of inconsistency risks. Whereas, occupancy, hot water demand, and all the building-related data could be implemented in the FMU of the associated house. Indeed, those data usually come from stochastic modeling (Baetens and Saelens 2016) and logically differ from one building to another. Three possibilities are available:

(1) create one FMU data-reader for all the buildings,

(2) create an FMU data-reader for each building, 
(3) embed data reading into each building's FMU.

The first solution is straightforward, but artificially creates dependencies between buildings where there is no need to. As a result, it introduces numerous communications that will affect computation time. Both the first and second options are partially incompatible with the boundary condition management of the IDEAS library, where boundary conditions are propagated inside each subcomponent using inner and outer models. Moreover, its introduce additional FMU, and concurrent computations (Vialle et al. 2017) with a severe complexity difference (reading values in a file vs. solving large scale ODE). Thus, those solutions would have introduced some structural modifications and would have produced a significant overhead.

Consequently, the third option was chosen, namely embedding data reading in each sub-component. The solution we developed automatically changes the resource path of each individual data reader during the instantiation of the FMU, by casting an integer parameter to the path's string. Code 2 shows an excerpt of the generic building model named GenBuigrid where the resource path is defined.

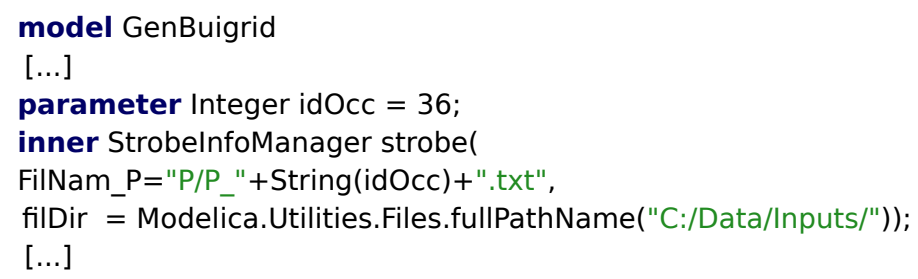

Code 2: Handling of the occupant resources in the building model

In this model, the inner instance strobe is a boundary condition manager dedicated to reading the occupancy profiles. The tunable parameter $i d O c c$ refers to the profile identification key. More information about StROBe (short for 'Stochastic Residential Occupant Behaviour') can be found in Baetens and Saelens (2016). In this particular example, the resource path points to $C: /$ data/Inputs $/ P / P$ _36.txt. This way, the resulting FMU is still generic, i.e. we can change the data profile by changing the parameter $i d O c c$, and the instantiation of the FMU only load one data profile, limiting the memory usage to the minimum.

\subsection{Scenarios}

This comparative study involves a detailed district energy system composed of a single low voltage distribution feeder supplying a variable number of building, $N_{b}$. More specifically, we consider 6 different cases, by setting $N_{b} \in\{3,6,9,12,24,48\}$. For each case, we implemented 6 comparative integration methods called scenarios. Those are based on 3 simulations and 3 co-simulations described in table 2 .

Simulation \#1 is the scenario that serves as reference for accuracy, and it represents the integrated simulation with the smallest integrator error tolerance of $10^{-8}$.

Simulation \#2 consists in a state-of-the-art setup, using a tolerance of $10^{-6}$, that is usually encountered in the literature (Protopapadaki and Saelens 2017; Protopapadaki, Baetens, and Saelens 2015).

Simulation \#3 uses a lower tolerance (of $10^{-3}$ ) in order to quantify speed-up and accuracy, and highlight the limits of the trade-off between tolerance and accuracy. 
Table 2. Description of the scenarios.

\begin{tabular}{lccc|ccc}
\hline & \multicolumn{3}{c|}{ Simulation } & \multicolumn{3}{c}{ Co-simulation } \\
\# label & $\log _{10}($ tol. $)$ & $\delta t(s)$ & solver & $\log _{10}($ FMItol. $)$ & $\delta t_{c o m}(s)$ & MA $^{*}$ \\
\hline- - 1 dym-sim-ref & -8 & 60 & Cvode & - & - & - \\
- 2 dym-sim-6 & -6 & 60 & Cvode & - & - & - \\
$*$ 3 dym-sim-3 & -3 & 60 & Cvode & - & - & - \\
+ - 4 dym-co-f120 & -6 & 120 & Cvode & -6 & 120 & Fixed \\
-5 dac-co-f120 & -6 & 120 & Cvode & -6 & 120 & Fixed \\
--6 dac-co-e60 & -6 & - & Cvode & -6 & $\in[60,900]$ & Euler \\
\hline$*$ Master-algorithm & & & & & &
\end{tabular}

Co-simulation \#4 uses the MA implemented in Dymola which uses a fixed communication step. The latter is fixed to $120 \mathrm{~s}$, small enough to capture the extrema of voltage and current quantities. This scenario will allow to assess the speed-up introduced by the co-simulation (without parallel computing).

Co-simulation \# $\mathbf{5}$ has the same setting as the scenario \#4 but is implemented in DACCOSIM, which allows parallel computing. This scenario will allow to assess the speed-up introduced by parallel computing.

Co-simulation \#6 is also implemented within DACCOSIM. The selected MA uses an adaptive communication time step controlled by one-step methods (Galtier et al. 2015).

With regard to the integration solver, only the Cvode algorithm is considered, both for the simulation and for the FMU exportation. It is a state-of-the-art variable step size algorithm for stiff systems. Although it may be interesting to use different integration algorithms for the sub-components, it is considered out of scope for the comparison in this paper.

\subsection{Key performance indicators}

The impact of the co-simulation is evaluated in terms of accuracy. To this end, a set of state-of-the-art simulation together with co-simulation scenarios has been defined and the comparison is realized based on root-mean-square error (RMSE), normalized root-mean-square error (NRMSE) and cross-correlation on three main quantities. These quantities, i.e. norm of the current $|I|$, voltage $|V|$ at the connection point and indoor temperature at the ground floor, are compared to the results of simulation \#1. RMSE, NRMSE and Cross-correlation are defined in eqs. (1) to (3).

$$
\begin{aligned}
\operatorname{RMSE}(\hat{y}, y) & =\sqrt{\frac{1}{n} \sum_{t}^{n}(\hat{y}(t)-y(t))^{2}} \\
\operatorname{NRMSE}(\hat{y}, y) & =\frac{\operatorname{RMSE}(\hat{y}, y)}{y_{\max }-y_{\min }} \\
(\hat{y} * y)(k) & =\sum_{t=1}^{n} \overline{\hat{y}}(t) y(t+k)
\end{aligned}
$$

where $\hat{y}$ and $y$ are the simulated values of the tested and reference simulation respectively, and $\overline{\hat{y}}$ denotes the conjugate of $\hat{y}$. Note that both sequences must be zero-padded for cross-correlation computation. $t$ and $k$ are respectively the time and the lag. In the 
following, we normalize the cross-correlation results by the auto-correlation of reference simulation $\# 1$ for $k=0$, such that :

$$
X_{\text {cor }}(k)=\frac{(\hat{y} * y)(k)}{(y * y)(0)}
$$

\section{Co-simulation performance}

In this paper, we propose a two-step approach of the accuracy and scalability analysis. First we will dedicate a detailed comparative study to the 3 -buildings case, i.e. $N_{b}=3$ in section 3.1. Then, scalability experiments will be presented for $N_{b} \in\{3,6,9,12,24,48\}$ in section 3.2 .

\subsection{Three grid-connected buildings case}

A first numerical experiment is conduced for a small amount of building in order to quantify the scalability of the co-simulation. To this end, simulation and co-simulation of three grid-connected buildings where conduced based on scenarios described in table 2 . For computational reasons, the following results consider one week horizon.
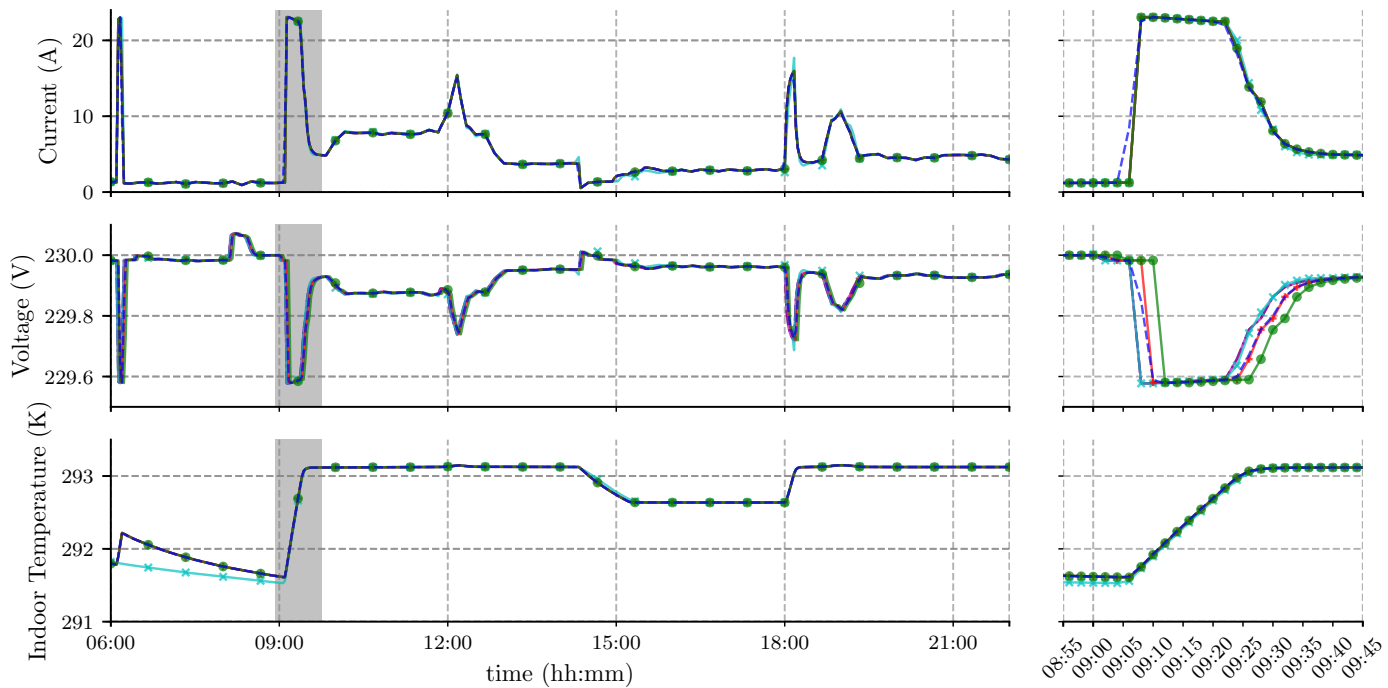

Figure 3. Dynamic simulation of the three grid-connected dwellings. On the left side, current, voltage and operational temperature of the first building are plotted for one day horizon. At the right side, the same quantities are displayed on a smaller time range (filled in gray on the left plots). - - 1 dym-sim-ref , — 2 dym-sim- $6, \nsucc 3$ dym-sim-3, +4 dym-co-f120, $\multimap 5$ dac-co-f120, - 6 dac-co-e60.

As a first result, plots of physical results are displayed on fig. 3 for one day time horizon. Current and voltage at the connection point, along with the operational temperature of the first floor zone, are displayed for the six simulation settings. To analyze the results on a closer range, one can refer to the right side plots. The heating system controls the inner temperature to follow the reference. The later is defined by the stochastic occupancy profile derived from StROBE (Baetens and Saelens 2016). In this case, one can see that the heating system is triggered in the morning and induces a hight 
power demand from $9 \mathrm{~h} 20$ to $9 \mathrm{~h} 45$. Differences between scenarios are not straightforward, mainly because we are focusing on numerical issues. But it is possible to pinpoint some deviations, principally on the voltage for the co-simulation scenarios (\#4,5 and 6) and on the low tolerance simulation (\#3). These deviations and delays will be analyzed and quantified in the following.

Table 3. Three grid-connected buildings case - Accuracy of scenarios defined in Table 2, with respect to the norm of the current, the voltage and indoor temperature at the ground floor for the first building.

\begin{tabular}{|c|c|c|c|c|c|c|}
\hline \# label & 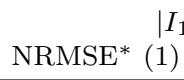 & ${ }^{1} \operatorname{RMSE}^{\dagger}(A)$ & $\begin{array}{r}\mid V \\
\operatorname{NRMSE}(1)\end{array}$ & RMSE $(V)$ & $\begin{array}{r}T O p \\
\text { NRMSE (1) }\end{array}$ & RMSE $\left({ }^{\circ} C\right)$ \\
\hline . - 1 dym-sim-ref & - & - & - & - & - & - \\
\hline-2 dym-sim- 6 & $7.83 \times 10^{-5}$ & $1.81 \times 10^{-3}$ & $6.98 \times 10^{-5}$ & $3.47 \times 10^{-5}$ & $3.63 \times 10^{-5}$ & $5.88 \times 10^{-5}$ \\
\hline$\star 3$ dym-sim-3 & $3.70 \times 10^{-2}$ & $8.56 \times 10^{-1}$ & $3.18 \times 10^{-2}$ & $1.58 \times 10^{-2}$ & $1.13 \times 10^{-1}$ & $1.83 \times 10^{-1}$ \\
\hline + 4 dym-co-f120 & $1.76 \times 10^{-2}$ & $4.08 \times 10^{-1}$ & $5.65 \times 10^{-2}$ & $2.81 \times 10^{-2}$ & $2.83 \times 10^{-3}$ & $4.58 \times 10^{-3}$ \\
\hline-5 dac-co-f120 & $1.76 \times 10^{-2}$ & $4.08 \times 10^{-1}$ & $5.65 \times 10^{-2}$ & $2.81 \times 10^{-2}$ & $2.83 \times 10^{-3}$ & $4.59 \times 10^{-3}$ \\
\hline - - 6 dac-co-e60 & $1.62 \times 10^{-2}$ & $3.76 \times 10^{-1}$ & $3.60 \times 10^{-2}$ & $1.79 \times 10^{-2}$ & $5.62 \times 10^{-3}$ & $9.12 \times 10^{-3}$ \\
\hline
\end{tabular}

Table 3 presents the main comparative results between scenarios in the case of $N_{b}=3$. Note that the CPU simulation time does not include compilation, FMU export nor (co)initialization elapse time for a fair comparison. In particular, RMSE and NRMSE are presented for the ground-floor operative temperature of the first building, and the norm of the current and voltage at that building's connection. CPU time results are included in table 4 for both absolute and relative simulation times. In terms of accuracy, one can see that simulation \#2 is highly accurate with respect to the reference, whereas simulation \#3 performs the worst and may not be suitable for DES assessments. It appears that co-simulations \#4 and \#5 don't yield significant differences, because fixed communication time step master-algorithms are similar in both Dymola and DACCOSIM. Finally, co-simulation \#6 provides the second most accurate results. In case of co-simulation scenarios, the lowest accuracy is observed for the voltage.

Table 4. Three grid-connected buildings case - CPU time and speed-up with respect to the scenarios

\begin{tabular}{r|cc}
\hline$\#$ & $\begin{array}{c}\text { CPU time* } \\
(\text { min, s) }\end{array}$ & $\begin{array}{l}\text { Speed-up } \\
(1)\end{array}$ \\
\hline$-\cdot 1$ & $(40,39)$ & 1 \\
-2 & $(21,43)$ & 1.88 \\
$* 3$ & $(16,28)$ & 2.50 \\
+4 & $(31,57)$ & 1.27 \\
-5 & $(21,05)$ & 2.38 \\
--6 & $(17,14)$ & 2 \\
$*$ * extrapolated CPU simulation time for one year horizon
\end{tabular}

In the following, the speed-up is defined as the ratio of the reference CPU time to the CPU time of the examined scenario. In table 4, we observe a small speed-up when comparing simulation to co-simulation scenarios using few buildings. Even in this particularly small case, the overhead introduced by the FMU communications are totally overcome by the co-simulation. From 3 dwellings, time savings are foreseen to be always positive. The effect of parallel computing can be highlighted comparing cosimulations \#4 and \#5. Indeed, we note a speed-up of 1.96/1.27 $\approx 1.54$ that can be directly linked to the implementation of the master algorithm in DACCOSIM. In this case, parallel computing introduces a speed-up of 1.54 (with dual core CPU). At this 
stage, simulation \#6 provides a good compromise between accuracy and computation time with a speed-up of 2.38 .

As RMSE and NRSME don't allow a fair comparison between delayed signals, we propose a cross-correlation analysis on fig. 4, based on the definition given in eq. (4). Figures 4(a) to 4(c) display normalized cross-correlation of the current, voltage and operative temperature with respect to the reference simulation \#1 as a function of displacement $k$. Peaks of the cross-correlation function with respect to the displacement denote a high dependence between signals. For $k=0$ (zero lag), the normalized crosscorrelation function shows the correlation between the 2 signals. The zoomed inset region displays this correlation for the results of the different scenarios compared to the reference. As expected, this value is close to 1 , with the larger deviation coming from simulation \#3. On the three physical quantities, one can note peaks at $k=0$ for the simulation scenarios (\#2 and \#3) and at $k=-120 s$ and $k=-60 s$ for co-simulation \#4, \#5 and \#6 that denotes a delay. In the detail of fig. 4(b), the delay on the voltage introduced by the co-simulation master algorithm (MA) can be observed. This is due to the fixed communication time step that delays the variable exchange. No similar delay is visible on current or temperature, however. We denote a general delay of $120 \mathrm{~s}$ for both scenarios \#4 and \#5, and about 60 s for co-simulation \#6, which correspond to the communication step time. Taking this delay into account, a cross-correlation up to 0.98 is reached for co-simulations \#4-6, whereas the cross-correlation for simulation \#3 is below 0.96. These results put into perspective the RMSE and NRMSE results of table 3, which don't take into account the delay introduced by the MA.

Delays on exchanged variables during DES simulations may be problematic for some situations. Hence, large communication time steps can incur errors, for control or event handling. Thus, a special attention must be paid for setting an adequate communication step size.

\subsection{Scalability analysis}

In the building simulation context, scalability is the capacity of a given simulation method or model to handle growing complexity. As stated in the literature review, this aspect is the main bottleneck of DES simulation. In this paper, the scalability analysis has been led on all scenarios defined in table 2 for a variable amount of grid-connected buildings, i.e. $N_{b} \in\{3,6,9,12,24,48\}$, on a one-week time horizon. Unfortunately, due to computational limits, results for $N_{b}=48$ are only available for the co-simulation scenarios \#4-6. At the time of the numerical experience, using Dymola 2017 and 8 Go of RAM, the integrated simulations \#1-3 are reaching memory limit for $N_{b}=48$. This is related to the known issues that we already described in the introduction. These issues may be remedied using more RAM or exploiting the high degree of sparsity of the jacobian and the hessien matrices to save memory (Casella 2015). But in the scope of this comparison, this was not addressed.

We first compare accuracy, in terms of RMSE and NRMSE, for all scenarios by means of box plots on fig. 5. On the x-axis, all scenarios are grouped with respect to the number of buildings. On the y-axis, using a logarithmic scale, one can see the errors of the main quantities, i.e. current, voltage and operative temperature. Boxes depict the group of all simulated buildings through their quartiles.

From fig. 5 it is possible to deduce a coherent trend for errors on voltage, where the RMSE appears to increase by one order of magnitude as the number of buildings grows to 24 . This is mainly explained by an increasing stress on the grid, leading to greater 
Postprint: V. Reinbold and C. Protopapadaki and J.-P. Tavella and D. Saelens. Assessing scalability of a low-voltage distribution grid co-simulation through functional mock-up interface, Journal of Building Performance Simulation, https://doi.org/10.1080/19401493.2019.1597923, 2019.

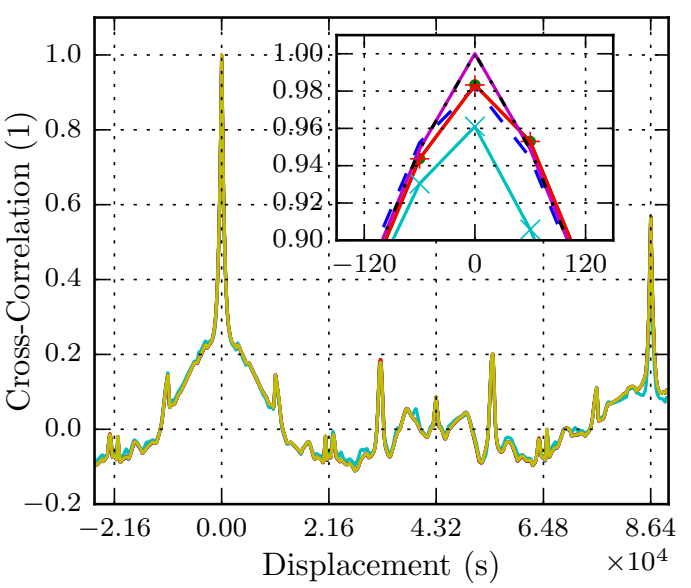

(a) Current $I_{1}$

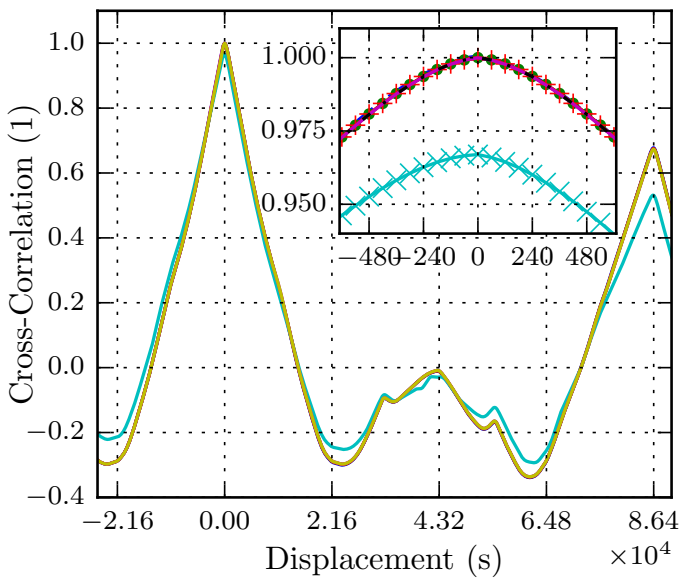

(c) Operational temperature $T O p_{1,1}$

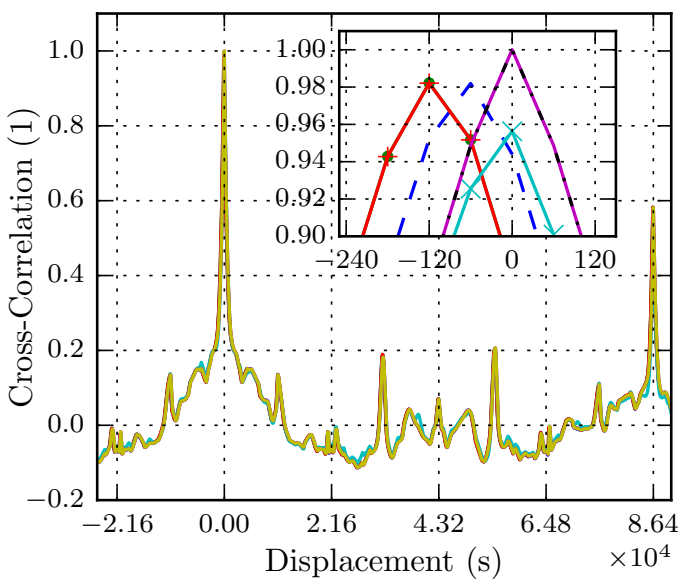

(b) Voltage $V_{1}$

Figure 4. Simulation of 3 grid connected buildings - Cross-correlation of the physical quantities with respect to the reference simulation for the first building. Insert plots are zooming on small range around zero. - - . $1 \mathrm{dym}-$ sim-ref,-2 dym-sim- $6, \nsucc 3$ dym-sim-3, † 4 dym-co-f120, $\rightarrow 5$ dac-co-f120, - 6 dac-co-e60. 
Figure 5. Box plot of the Root Mean Square Error (RMSE) and Normalized RMSE (NRMSE) for the main physical quantities with respect to the reference simulation and grouped by number of buildings.
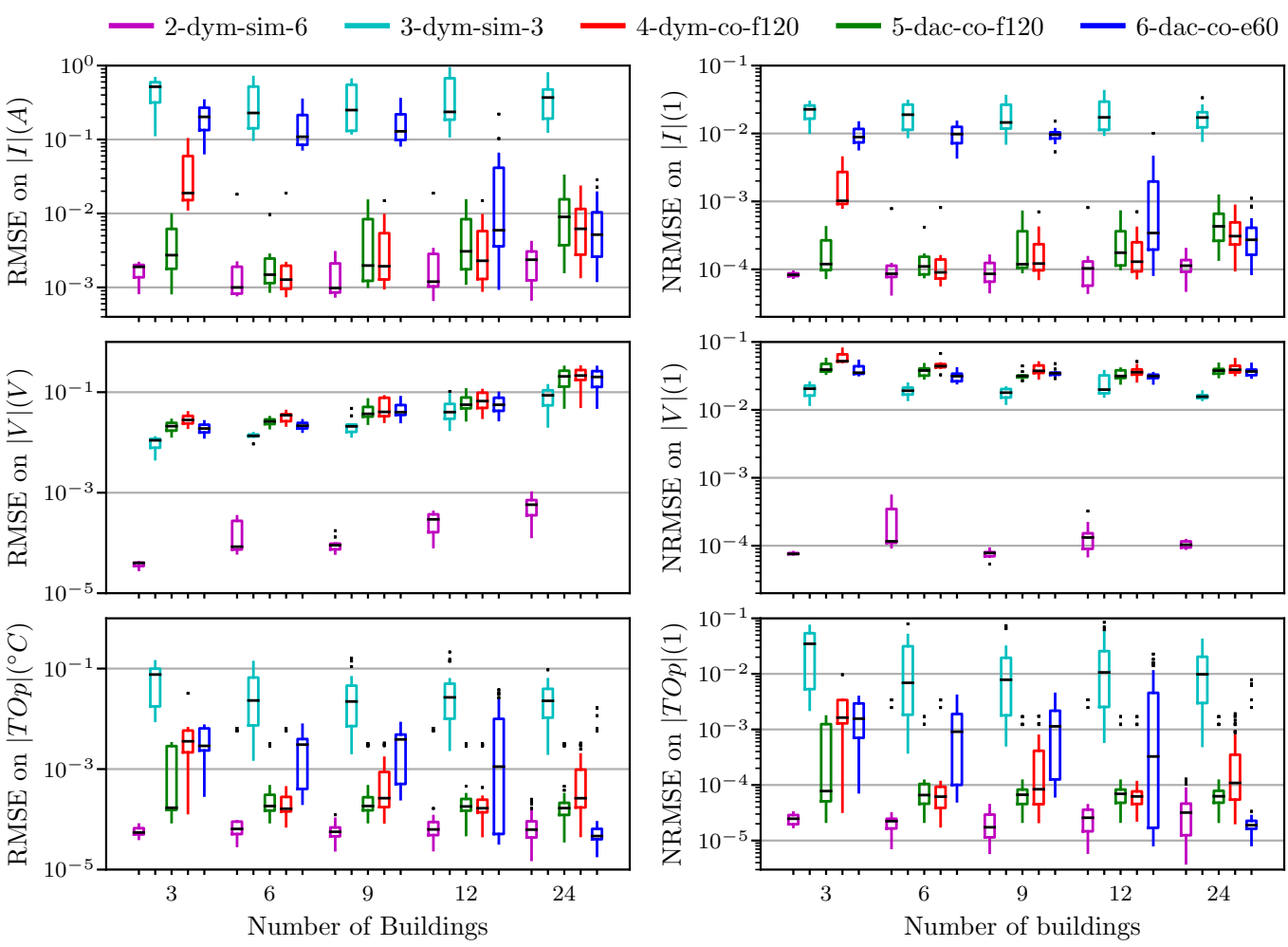

variation in the voltage. As such, the NRMSE remains relatively constant because the range of voltage $V_{\max }-V_{\min }$ increase as well. For more buildings, if the cable is sized appropriately to handle the loads, co-simulation is not expected to introduce important errors. Co-simulation \#6 results in higher error than scenarios \#2 and \#4-5, about 2 order of magnitude for the current and $N_{b} \in\{3,6,12\}$, for instance. This is actually surprising, since it uses a smaller communication time step than co-simulations \#4-5. One could explain this difference by the error that re-sampling introduces, given the variable communication step size.

The computation time comparison between scenarios with respect to the number of buildings is shown on fig. 6. In this figure, we plot the computation time and the speed-up with respect to the reference simulation, where both, $x$ and $y$ axes are in logarithmic scale. Computational time includes elapsed time during initialization and integration for both simulation and co-simulation scenarios. For all scenarios, elapsed time during (co)-initialization is between 1 and $8 \mathrm{~s}$, which represent less than $1 \%$ of the total computational time for two weeks time horizon. The computation time does not consider compilation. The latter can be significant for integrated scenarios because the compiler used in Dymola does not exploit repetitive structures. For co-simulation approach however, the modeler can easily export one generic FMU and instantiate it several times. This effect can be a significant advantage for co-simulation approach, when the model involves numerous repetitive structures.

Speed-up calculations are not available for $N_{b}=48$, because the reference simulation couldn't be carried out, due to memory limitations.

With regard to the integrated simulations, we note a linear increase in simulation 


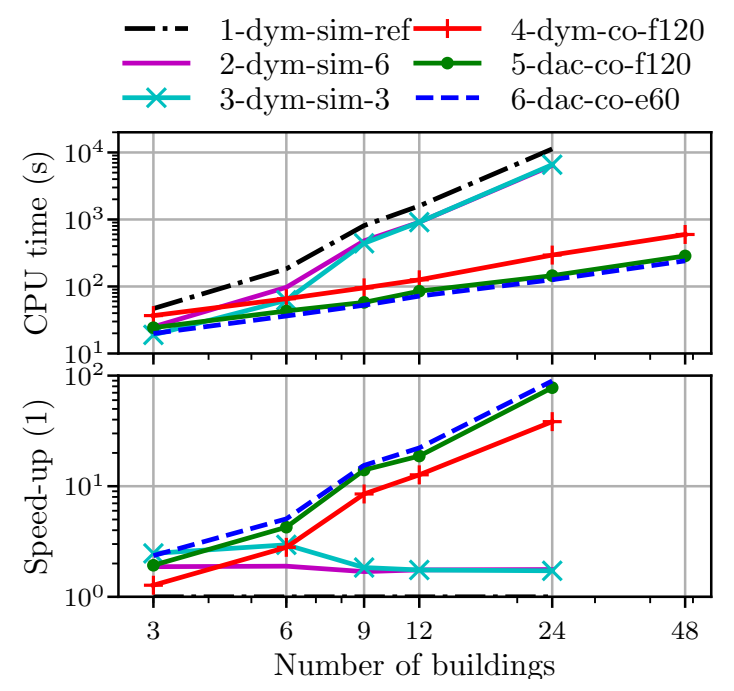

Figure 6. CPU time and speed-up for one week time horizon

time with respect to $N_{b}$. For $N_{b}=24$, simulations \#1-3 are particularly slow, requiring respectively $3 \mathrm{~h} 08,1 \mathrm{~h} 47$ and $1 \mathrm{~h} 49$ for a week-long simulation. Differences in computation time between these simulations can mainly be attributed to the difference in tolerance. Speed-up of about 2.0 can be achieved when decreasing the error tolerance for more than 9 buildings. However, it is not straightforward to explain why the CPU time becomes similar for simulations \#2 and \#3. Memory writing and reading may become predominant in this case, such that it is not possible to spot a significant difference.

Co-simulations \#4-6 also display a linear behavior with respect to $N_{b}$, but with better scalability performance. Compared to the reference simulation, the speed-up is linearly and ranges from 2.22 for $N_{b}=3$, to about 90 for $N_{b}=24$. Based on those results, one could extrapolate speed-up for a number of dwellings higher than 24.

Among co-simulations, scenario \#6, using parallel computing and adaptive communication step size, has the best scalability performance. Scenario \#5 offers a fairly constant speed-up around 1.5-2 compared to \#4. This speed-up could be attributed to the DACCOSIM implementation, which uses a parallel computing scheme.

\section{Conclusion}

This article quantifies the scalability of co-simulation algorithms in the context of district energy systems. First, it tackles practical aspects of co-simulation of DES using the Modelica language and FMI standard. It involves decomposition, adaptation for FMU components and handling of input data. In particular, we introduce a method to deal with external data inputs using casting to generate heterogeneous occupancy data and save memory during the FMU instantiation.

Secondly, to analyze the possibilities that co-simulation offers, a comparative study involving 3 to 48 grid-connected buildings, heterogeneous input data, different masteralgorithms and two co-simulation environments has been performed. This comparison not only determines the reduction in CPU time, but also evaluates the accuracy for the main outputs. Co-simulation shows a better scalability compared to simulations for 3 to 48 buildings and the computation time seems proportional to the amount of buildings. 
Moreover, we note a significant time saving using parallel computing: CPU usage is divided by a factor of about 2.0 using dual-core CPU. It cannot be directly attributed to the co-simulation method itself, but could be considered as a positive side effect, since co-simulation facilitates the implementation of parallel computing. This study also highlights the advantages of the adaptive communication step size algorithm implemented in DACCOSIM, which shows good scalability results while ensuring good accuracy. Globally, we note a higher performance of the DACCOSIM master algorithm than the Dymola 2017 implementation. At the same time, co-simulations maintain a good level of accuracy for the main quantities of interest, namely current, voltage and indoor temperature. Nevertheless, attention should be paid to the small delay introduced by co-simulation algorithms, which might influence control.

Within the limits of the studied case in this paper, co-simulation has proven to significantly mitigate scalability issues of district energy systems, reaching a speed-up of 90 for a 24-dwellings district. Moreover, the speed-up is quickly increasing with the number of dwellings which indicates good performance can be expected also for larger DES.

Co-simulation for DES offers many future work opportunities. In terms of methodology, parallel computing and superFMU - an FMU containing inner co-simulation and parallel computing schemes - seem promising in terms of CPU usage and interoperability. Moreover, deeper studies on the impact of decomposition are required, especially on the building level where different dynamics and controls are involved. Finally, a wider spectrum of test cases would give more insight into scalability of realistic DES co-simulation. For instance, cases with heterogeneous building structures and systems could be considered.

\section{Acknowledgment}

This work has been conducted within the EFRO-SALK project, which receives the support of the European Union, the European Regional Development Fund, Flanders Innovation \& Entrepreneurship and the Province of Limburg.

\section{References}

Baetens, Ruben. 2015. "On externalities of heat pump-based low-energy dwellings at the lowvoltage distribution grid." PhD diss., KU Leuven.

Baetens, Ruben, Roel De Coninck, Filip Jorissen, Damien Picard, Lieve Helsen, and Dirk Saelens. 2015. "OpenIDEAS - an open framework for integrated district energy simulations." In 14th Conference of International Building Performance Simulation Association, .

Baetens, Ruben, and Dirk Saelens. 2016. "Modelling uncertainty in district energy simulations by stochastic residential occupant behaviour." Journal of Building Performance Simulation 9 (4): 431-447. http://dx.doi.org/10.1080/19401493.2015.1070203.

Blochwitz, Torsten, Martin Otter, Martin Arnold, Constanze Bausch, H Elmqvist, A Junghanns, J Mauß, et al. 2011. "The functional mockup interface for tool independent exchange of simulation models." In Proceedings of the 8th International Modelica Conference; March 20th-22nd; Technical Univeristy; Dresden; Germany, 105-114. Linköping University Electronic Press.

Carvalho, LF, J Ignacio Torrens, JLM Hensen, L Xu, and GC Graça. 2016. District Heating Systems. Technical Report. Eindhoven University of Technology and University of Lisbon.

Casella, Francesco. 2015. "Simulation of large-scale models in modelica: State of the art and fu- 
ture perspectives." In Proceedings of the 11th International Modelica Conference, Versailles, France, September 21-23, 2015, 459-468. Linköping University Electronic Press.

De Coninck, Roel, Fredrik Magnusson, Johan Åkesson, and Lieve Helsen. 2015. "Toolbox for development and validation of grey-box building models for forecasting and control." Journal of Building Performance Simulation 1-16.

European Commision. 2016. Communication on Clean Energy For All Europeans. Technical Report. European Commision. http://eur-lex. europa.eu/resource.html?uri=cellar: fa6ea15b-b7b0-11e6-9e3c-01aa75ed71a1.0001.02/DOC_1\&format=PDF.

European Union. 2012. Directive 2012/27/EU of the European Parliament and of the Council of 25 October 2012 on energy efficiency, amending Directives 2009/125/EC and 2010/30/EU and repealing Directives 2004/8/EC and 2006/32/EC Text with EEA relevance. Technical Report. European Union. http://eur-lex.europa.eu/legal-content/EN/TXT/?qid= $1399375464230 \&$ uri=CELEX : 32012L0027.

Frederiksen, Svend, and Sven Werner. 2013. District heating and cooling. Studentlitteratur.

Fritzson, Peter. 2015. Principles of Object-Oriented Modeling and Simulation with Modelica 3.3. John Wiley \& Sons.

Galtier, Virginie, Stephane Vialle, Cherifa Dad, Jean-Philippe Tavella, Jean-Philippe Lam-YeeMui, and Gilles Plessis. 2015. "FMI-based distributed multi-simulation with DACCOSIM." In Proceedings of the Symposium on Theory of Modeling 85 Simulation: DEVS Integrative MESS Symposium, 39-46. Society for Computer Simulation International.

IDEAS. 2017. https://github.com/open-ideas/IDEAS/tree/v1.0.0.

Jensen, Søren Østergaard. 1995. "Validation of building energy simulation programs: a methodology." Energy and buildings 22 (2): 133-144.

Kersken, M, I Heusler, and P Strachan. 2014. "Full Scale Empirical Validation for Building Energy Simulation Programs." In Liège: 9th International Conference on System Simulation in Buildings, .

Kim, Eui-Jong, Gilles Plessis, Jean-Luc Hubert, and Jean-Jacques Roux. 2014. "Urban energy simulation: Simplification and reduction of building envelope models." Energy and Buildings 84: $193-202$.

Madsen, Henrik. 2007. Time series analysis. CRC Press.

Melgaard, Henrik. 1994. "Identification of physical models." PhD diss., Technical University of Denmark, Department of Informatics and Mathematical.

Müller, D, M Lauster, A Constantin, M Fuchs, and P Remmen. 2016. "AixLib-An Open-Source Modelica Library within the IEA-EBC Annex 60 Framework." BauSIM 2016 3-9.

Nouidui, Thierry, Michael Wetter, and Wangda Zuo. 2014. "Functional mock-up unit for cosimulation import in EnergyPlus." Journal of Building Performance Simulation 7 (3): 192202.

Nouidui, Thierry Stephane, Kaustubh Phalak, Wangda Zuo, and Michael Wetter. 2012. "Validation and application of the room model of the Modelica Buildings library." In Proceedings of the 9th International MODELICA Conference; September 3-5; 2012; Munich; Germany, 727-736. Linköping University Electronic Press.

Nytsch-Geusen, Christoph, and Werner Kaul. 2015. "GENERATION OF DYNAMIC ENERGETIC DISTRICT MODELS FROM STATISTICAL RELATIONSHIPS.” In 14th Conference of International Building Performance Simulation Association, Hyderabad, India, Dec. $7-9$, .

Privara, Samuel, Jiří Cigler, Zdeněk Váňa, Frauke Oldewurtel, Carina Sagerschnig, and Eva Žáčeková. 2013. "Building modeling as a crucial part for building predictive control." Energy and Buildings 56: 8-22.

Protopapadaki, Christina, Ruben Baetens, and Dirk Saelens. 2015. "Exploring the impact of heat pump-based dwelling design on the low-voltage distribution grid." In 14th Conference of International Building Performance Simulation Association, .

Protopapadaki, Christina, and Dirk Saelens. 2017. "Heat pump and PV impact on residential low-voltage distribution grids as a function of building and district properties." Applied Energy 192: 268-281. 
Ptolemaeus, Claudius, ed. 2014. System Design, Modeling, and Simulation using Ptolemy II. Ptolemy.org. http://ptolemy.org/books/Systems.

Raad, A., v. Reinbold, B. Delinchant, and F. Wurtz. 2015. "FMU software component orchestration strategies for co-simulation of building energy systems." In Technological Advances in Electrical, Electronics and Computer Engineering (TAEECE), 2015 Third International Conference on, April, 7-11.

Reinhart, Christoph F, and Carlos Cerezo Davila. 2016. "Urban building energy modeling-A review of a nascent field." Building and Environment 97: 196-202.

Robinson, Darren, Frédéric Haldi, J Kämpf, Philippe Leroux, Diane Perez, Adil Rasheed, and Urs Wilke. 2009. "CitySim: Comprehensive micro-simulation of resource flows for sustainable urban planning." In Proc. Building Simulation, 1614-1627.

Saelens, Dirk, Michael Wetter, and Christoph van Treeck. 2017. "ANNEX 60: International Tool development for Advanced Energy Performance Simulations." In Proceedings of the EnergieWendeBauen Kongress 2017, 272-276.

Verbruggen, Bart, Juan Van Roy, Roel De Coninck, Ruben Baetens, Lieve Helsen, and Johan Driesen. 2011. "Object-oriented electrical grid and photovoltaic system modelling in Modelica." In Proceedings of the 8th International Modelica Conference; March 20th-22nd; Technical Univeristy; Dresden; Germany, 730-738. Linköping University Electronic Press.

Vialle, S., J.-P. Tavella, C. Dad, R. Corniglion, M. Caujolle, and Vincent. Reinbold. 2017. "Scaling FMI-CS Based Multi-Simulation till Thousand FMUs on Infiniband Cluster." In 12th International Modelica Conference, May 15-17, 2017, Prague, Czech Republic, .

Walter, Emmanuel, and Jérôme Henri Kämpf. 2015. "A verification of CitySim results using the BESTEST and monitored consumption values." In Proceedings of the 2nd Building Simulation Applications conference, 215-222. Bozen-Bolzano University Press.

Wetter, Michael. 2011. "Co-simulation of building energy and control systems with the Building Controls Virtual Test Bed." Journal of Building Performance Simulation 4 (3): 185-203.

Wetter, Michael, Marco Bonvini, and Thierry S. Nouidui. 2016. "Equation-based languages - A new paradigm for building energy modeling, simulation and optimization." Energy and Buildings 117: 290 - 300. http://www.sciencedirect.com/science/article/pii/ S0378778815303315.

Wetter, Michael, Marcus Fuchs, Pavel Grozman, Lieve Helsen, Filip Jorissen, Moritz Lauster, Dirk Müller, et al. 2015. "IEA EBC Annex 60 Modelica library-an international collaboration to develop a free open-source model library for buildings and community energy systems." In Building simulation, .

Widl, Edmund, Wolfgang Müller, Atiyah Elsheikh, Matthias Hörtenhuber, and Peter Palensky. 2013. "The FMI++ library: A high-level utility package for FMI for model exchange." In Modeling and Simulation of Cyber-Physical Energy Systems (MSCPES), 2013 Workshop on, 1-6. IEEE.

Zhang, Kun, Narges Roofigari E., Humberto Quintana, and Michaël Kummert. 2015. "Assessing simplified and detailed models for predictive control of space heating in homes." In Proceedings of the Ninth International Conference,

Zucker, Gerhard, Florian Judex, Max Blöchle, Mario Köstl, Edmund Widl, Stefan Hauer, Aurelien Bres, and Jyoti Zeilinger. 2016. "A new method for optimizing operation of large neighborhoods of buildings using thermal simulation." Energy and Buildings 125: 153 - 160. http://www.sciencedirect.com/science/article/pii/S0378778816303589. 